\title{
Influence of comfort and social stimuli on a comfort movement and a display derived from it
}

\author{
PETER SEBBEL, HEINZ DÜTTMANN\& TON GROOTHUIS \\ Department of B ehavioural Biology, U niversity of Groningen \\ ( Received 20 J une 1996; initial acceptance 2 A ugust 1996; \\ final acceptance 11 M arch 1997; M S. number: 5272R)
}

\begin{abstract}
A bstract. W e tested the extent of emancipation of the W histle-shake display of male shelducks, T adorna tadorna, from causal factors controlling its presumed evolutionary precursor, the Body-shake, a comfort movement. Both motor patterns show similarities in form and alternate in a yearly rhythm. First, in an artificial rain experiment, we analysed the influence of a stimulus controlling comfort movements. In spring almost exclusively Whistle-shakes were induced even in the absence of social stimuli, while in summer the birds performed predominantly Body-shakes. This is probably related to the seasonal production of sex hormones. Second, we tested the influence of social stimuli by confronting shelducks with displaying and non-displaying males. During moult when males almost exclusively perform Body-shakes we found no influence of social stimulation on the frequency or form of shakes. However, an effect of social stimulation was found in a period when the drakes gradually replace the Body-shake by the Whistle-shake which is after moult in autumn. In this experiment, birds were confronted with either an empty cage (control), artificial rain, a non-displaying or a displaying male conspecific. In relation to the control situation the birds performed more Whistle-shakes when stimulated socially and more Body-shakes when stimulated with artificial rain. The extent to which both shaking patterns share the same causal factors is discussed.
\end{abstract}

(C) 1998 The A ssociation for the Study of A nimal Behaviour

In ethology it is generally accepted that during the course of evolution a social display can become independent of the causal factors controlling its precursor patterns (Tinbergen 1952; Baerends 1975). A lthough the degree of this emancipation can give valuable information on the evolution and proximate causation of displays, it has not been the subject of much experimental testing. Recently D üttmann \& G roothuis (1996) compared the proximate causation of the Whistleshake display of male shelducks with that of its evolutionary precursor, the comfort movement of Body-shaking. They found that males perform both the Whistle-shake and the Body-shake after wetting their plumage. This suggests an

Correspondence and present address: P. Sebbel, Institut für Molekularbiologie und Biophysik, ETH Hönggerberg, HPM E 13, 8093 Zürich, Switzerland (email: sebbel@mol.biol.ethz.ch). H. D üttmann and T. Groothuis are at the Department of Behavioural Biology, University of Groningen, P.O. Box 14, 9750 A A $\mathrm{H}$ aren, The $\mathrm{N}$ etherlands. influence of comfort stimuli on the occurrence of the display pattern. Consequently, Düttman \& $G$ roothuis concluded that the Whistle-shake is not emancipated from the causal factor for its evolutionary precursor, the Body-shake. H owever, their comparison of the causation of both motor patterns was incomplete because: (1) these birds were tested when housed in groups of conspecifics and, therefore, the influence of social stimuli was not excluded; (2) the Whistle-shake appeared to be sensitive to social stimulation but the sensitivity of the Body-shake for social stimuli was not tested; (3) the experiments were carried out either in spring when the W histle-shake predominates or in summer when the Body-shake is almost exclusively performed. Therefore the results may be confounded by an influence of season. In summary, these reasons make it impossible to draw a conclusion about the extent to which the shaking patterns differ in their causation. Therefore our aim in this paper was (1) to test whether the Whistle-shake can be triggered by comfort stimuli 
(a)

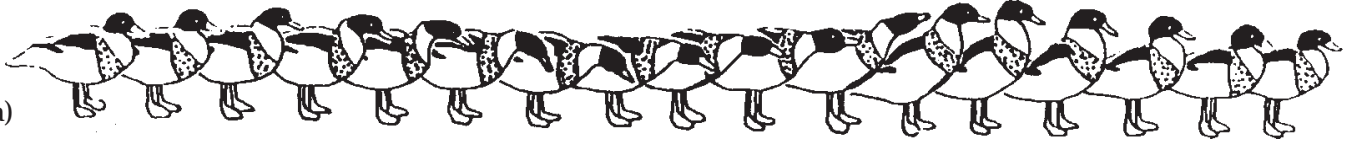

(b)

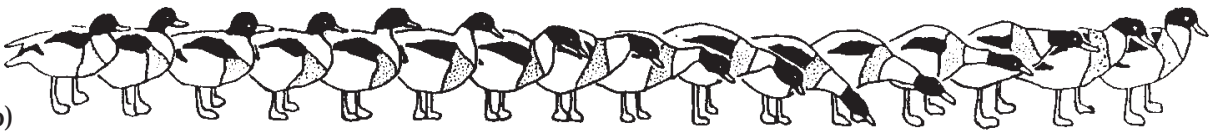

(c)

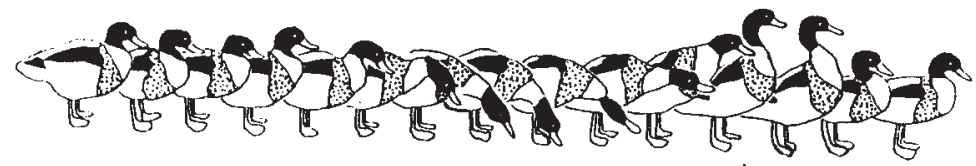

(d)

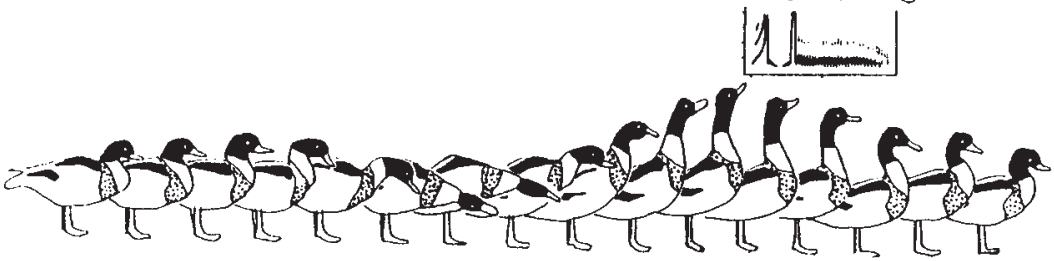

Figure 1. F orm of shaking patterns of adult male shelducks: (a) Body-shake; (b) D eep Intermediate form; (c) High Intermediate form; (d) Whistle-shake with trill (inset). M odified after R atermann et al. (1990).

only, (2) to test whether the Body-shake can be triggered by social stimuli, and (3) to compare the influence of comfort and social stimuli on the Whistle-shake and the Body-shake more directly during the period in which both patterns are simultaneously present in the repertoire.

The Body-shake is a typical comfort movement in all A natidae. It removes water from the plumage and rearranges the body feathers (M CK inney 1965). The Whistle-shake is a display given by males during courtship and territorial interactions. In ontogeny the Whistle-shake emerges gradually via intermediate forms from the Bodyshake (D üttmann 1992). In the display all shaking components are unchanged, except for the last part of the movement. Instead of taking back the head directly, a bill-tipping component is added while the drake utters a trill (F ig. 1; see D üttmann \& Groothuis 1996). Body-shake and Whistleshake alternate in a yearly rhythm: the Whistleshake is performed almost exclusively for most of the year. During moult in summer, however, sheldrakes perform the Body-shake, never the Whistle-shake display. D uring a couple of weeks before and after moult all shaking patterns are present in one bird including intermediate forms ( $R$ atermann et al. 1990; R atermann 1991). The clear relationship between the Body-shake and the Whistle-shake, and the fact that comfort stimuli are relatively easy to manipulate, make these motor patterns an excellent model to study the extent of emancipation in social displays.

\section{EXPERIMENT I: ARTIFICIAL RAIN EXPERIMENTS IN SPRING AND SUM MER}

This experiment was designed to investigate to what extent the display has been emancipated from one of its presumed original causal factors. In particular, we tested whether the Whistleshake is sensitive to a comfort stimulus relevant for the Body-shake, even in a situation where social factors are absent. Therefore we carried out artificial rain experiments with socially isolated birds. To compare the results for the Whistleshake with data from the Body-shake, we did similar experiments in A pril when birds predominantly perform Whistle-shakes and in J une when the Body-shake is almost exclusively present. 


\section{$M$ ethods}

F or both experiments in A pril and J une we used the same five male shelducks. They were obtained from professional breeders and were at least 2 years of age. A round the time of the experiments they were housed individually in visual isolation from other ducks in outdoor aviaries that included a $0.75 \mathrm{~m}^{2}$ water basin. The aviaries measured $1.5 \times 3 \times 2.5 \mathrm{~m}$ high in all experiments. The water was refreshed almost daily. The animals were fed ad libitum with grain and pellets as used in duck and geese farms.

In the tests we sprayed the birds with water from a hide next to the aviary 10 times in succession for $1 \mathrm{~s}$ each $5 \mathrm{~min}$ to release shaking patterns. We recorded the behaviour of the birds on videotape from a hide $5 \mathrm{~m}$ away.

We classified the different forms of shaking on the basis of the amplitude of the head movement when the head is taken back after the completion of the head-neck-shaking component. In the Body-shake the head is taken back directly to the normal relaxed position. In the Whistle-shake the head is raised until the neck is stretched vertically and the bill is pointing upwards. The movement is accompanied by a whistling trill. In the $\mathrm{H}$ igh Intermediate-shake the neck is stretched while the bill is not raised above the horizontal line. In the D eep Intermediate-shake the neck is stretched less extensively than in the two previous forms (F ig. 1).

\section{R esults}

In both spring and summer the isolated males responded in nearly $100 \%$ of the sprays with a shaking pattern (Fig. 2). A paired t-test did not show a difference between the seasons in this respect $\left(t_{4}=3.07\right.$, Ns). H owever, there was a clear effect of season on the type of the shaking response. In spring all males reacted exclusively with Whistle-shakes, whereas in summer only Body-shakes and D eep Intermediate forms were elicited by artificial rain stimuli (Fig. 2). The difference in the frequency of Whistle-shakes between periods was highly significant (paired t-test: $\left.t_{4}=31.62, P<0.001\right)$. During the tests in both periods none of the isolated males was ever seen to perform other social displays. In conclusion, the Whistle-shake display is still under the control of factors affecting plumage condition and influencing the performance of the Body-shake.

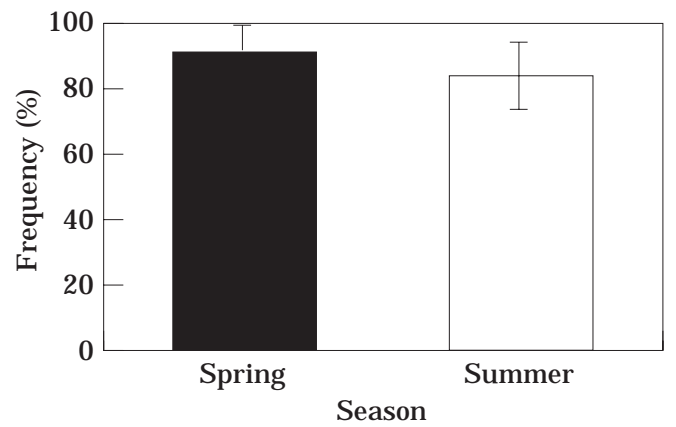

Figure 2. Percentages of artificial rain stimuli in spring and summer in which shaking responses ( $\bar{X} \pm \mathrm{SE})$ occurred. D ata are from five isolated adult sheldrakes. 口: Whistle-shake; $\square$ : D eep Intermediate-shake and Body-shake.

\section{EXPERIMENT II: SOCIAL STIMULATION IN SUMMER}

In spring the Whistle-shake is, in addition to comfort stimuli, sensitive to social stimulation (Düttmann \& Groothuis 1996). The possible influence of social factors on the Body-shake is, however, still unknown. Therefore we confronted adult males with different social stimuli in summer, when they predominantly perform the Bodyshake.

\section{M ethods}

During moult in summer nine shelduck pairs were housed separately in rows of three adjacent aviaries as described above (experiment I). W ithin a row the aviaries were separated by wire-netting, so that the birds could see each other but did not establish a rank order. Birds of one row could not see the birds in another row. In each row the male in the middle was the stimulus bird that should stimulate the test males to the left and the right. To obtain an actively displaying stimulus bird it was implanted with testosterone propionate. We applied $30 \mathrm{mg}$ of the hormone in silicon tubes (inner diameter $=1 \mathrm{~mm}$, outer diameter $=3 \mathrm{~mm}$, length $3.5 \mathrm{~cm}$ ), which we implanted subcutaneously in the neck using lidocaine as local anaesthetic. Because the effect of the hormone on behaviour develops gradually within about 4 days, we started observations 4 days after implantation. A s the control, the same test birds, housed in the same cages, were confronted with an untreated 
male in the middle cage. We tested six pairs in this way but analysed only the data of the males. F our pairs were confronted with the implanted stimulus bird first. Thereafter we had to exchange the stimulus males since they continued to display after we removed the hormone-filled tubes and therefore could not be used as stimulus birds in the control phase. The other two pairs were first observed in the control situation and subsequently confronted with the same, now implanted, stimulus birds in the experimental situation. The control as well as the experimental period lasted 2 weeks.

We observed all birds $10 \mathrm{~h}$ a week from a hide $5 \mathrm{~m}$ away. Observations took place between 0900 and 1200 hours and between 1300 and 1700 hours. D uring these hours we observed two test males of the same row simultaneously and recorded the different forms of shaking behaviour (see experiment I). The frequency of shaking was calculated for every week separately to control for an effect of set-up changes.

A ll data were $\log (x+1)$ transformed to achieve a normal distribution. For statistical analyses we carried out a three-factor MANOVA, with repeated measures for all factors. One factor represented the form of the shaking patterns (four levels), the second factor the experimental condition (two levels) and the last factor the week of the control or test phase (two levels). We tested for the effects of stimulus condition and week and for the interaction effects of shaking form $\times$ stimulus condition and shaking form $\times$ week. In all these cases df within cells was 5 . A dditionally, we performed power analyses for the tests at the 0.05 level of significance and with an $80 \%$ chance of detecting differences between the means.

\section{E thical note}

Experiments II and III (see below) involved the application of testosterone proprionate to sexually inactivate male shelducks. Both experiments were carried out in line with the $D$ utch legal require ments for animal experiments and in line with the ASA B guidelines for the use of animals in research. By restricting the hormonal treatment to only 3 weeks and by using implants that give plasma testosterone levels within the physiological range of adult males, we reduced the impact of the hormone which did not significantly affect the birds' welfare. F urthermore we treated only four individuals which we used as displaying stimulus birds. All birds became normally reproductive in the next breeding season.

\section{R esults}

The total frequencies of all shaking patterns in the test males did not differ significantly between the stimulus conditions (effect of stimulus condition: $F_{1,5}=0.33$, Ns; effect of week: $F_{1,5}=2.22, \mathrm{Ns}$ ). Whistle-shakes were hardly performed at all and with regard to the form of shaking we could not find differences between the test situations ( $F$ ig. 3; interaction effect of form $\times$ stimulus condition: $F_{3,15}=0.74$, Ns). There was also no significant effect of the set-up changes (interaction effect of form $\times$ week: $F_{3,15}=1.92$, Ns). A power analysis revealed that detectable differences between the groups were very small, often less than $10 \%$ of the average observed frequencies. (Body-shake: detectable difference: 0.044 , observed difference: 0.0074; D eep I ntermediate form: detectable difference: 0.136 , observed difference: 0.0082 ; W histleshake: detectable difference: 0.350 , observed difference: 0.014$)$. The $\mathrm{H}$ igh Intermediate form was the only exception ( $N=6$, detectable difference: 0.032 , observed difference: 0.1063 ).

In conclusion social stimulation does not influence the frequency of the Body-shake. So, although the Whistle-shake is sensitive to comfort stimulation in spring as the Body-shake is in summer (experiment I), the Body-shake is not sensitive to social stimulation in summer (experiment II), in contrast to the Whistle-shake in spring (D üttmann \& G roothuis 1996). O wing to a possible strong effect of season, constraining the performance of W histle-shakes in summer, the influence of social stimulation on the Whistle-shake could not be assessed reliably during this period.

\section{EXPERIMENT III: SOCIAL STIMULATION AND ARTIFICIAL RAIN IN AUTUM N}

The previous experiments were done at the times of year when birds show almost exclusively either the W histle-shake or the Body-shake. To compare the causation of both motor patterns more directly we tried to assess the influence of artificial rain and social stimulation in a period during which all shaking patterns are present in one bird. 


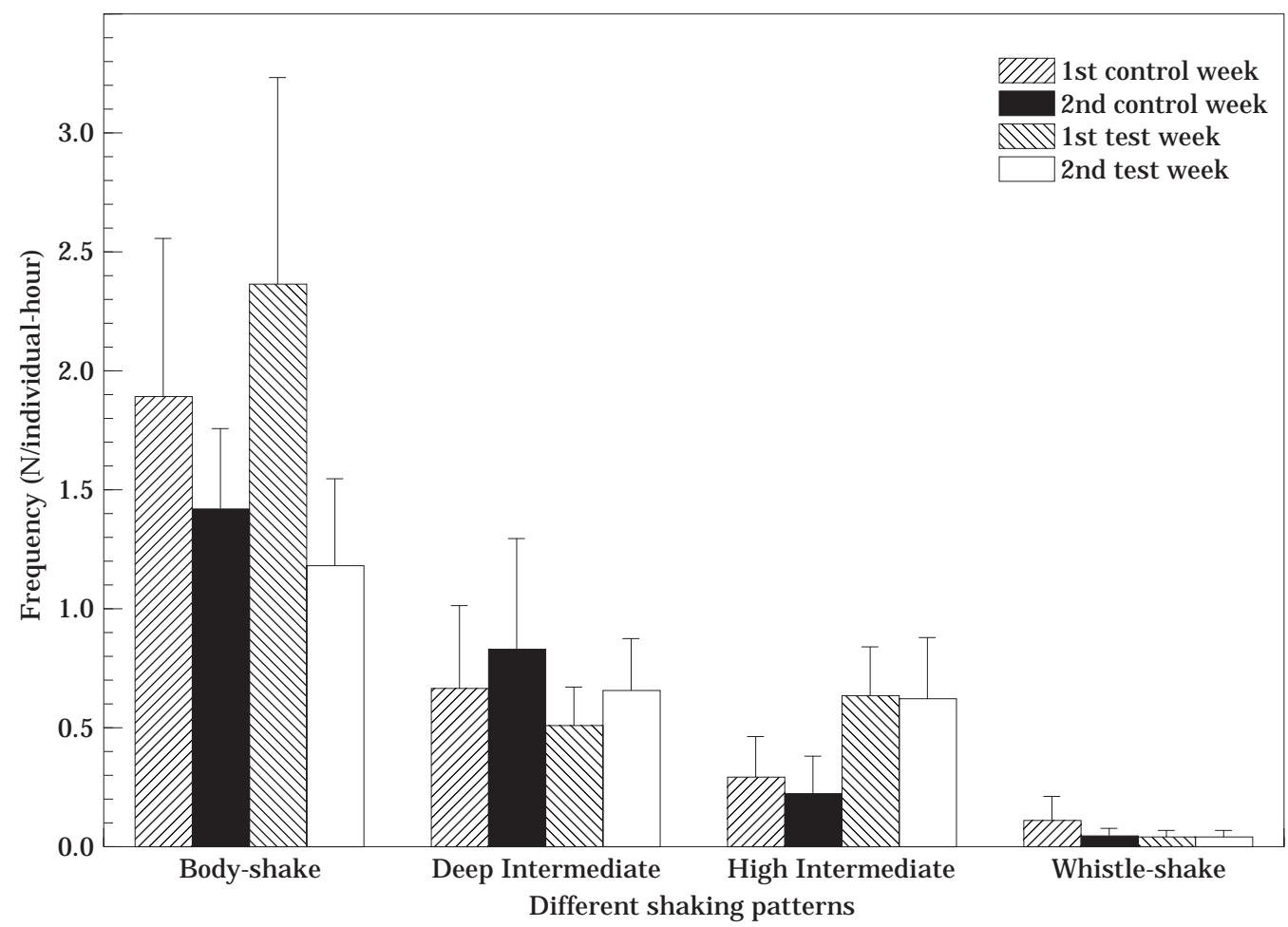

Figure 3. F requencies $(\bar{X}+\mathrm{sE})$ per $\mathrm{h}$ of different shaking patterns of six male shelducks, which were confronted with a sexually inactive shelduck pair (control weeks) and an actively displaying pair (test weeks).

\section{M ethods}

We tested adult male shelducks under five conditions: (1) control (no stimulation); (2) weak comfort stimulation (1 s artificial rain); (3) strong comfort stimulation (10 s artificial rain); (4) weak social stimulation (confrontation with an untreated male in the adjacent cage); (5) strong social stimulation (confrontation with a testosterone propionate-treated, displaying male in the adjacent cage).

All five tests lasted for $100 \mathrm{~min}$. In the control situation we observed the birds in the experimental cage (see below) without any stimulation. F or conditions 2 and 3 we sprayed all birds with water 10 times for 1 and $10 \mathrm{~s}$, respectively, at 10-min intervals in the experimental cage. $F$ or conditions 4 and 5 the birds were confronted with another male, presented in the cage next to them. This adjacent cage was similar to the experimental cage and separated from it by wire-netting. In condition 5 the stimulus bird was implanted with testosterone propionate (see experiment II for details), whereas in condition 4 we used a bird in the same phase of the yearly rhythm as the tested birds. To induce shaking-patterns in the stimulus birds, we showered them 10 times at $10-\mathrm{min}$ intervals for $1 \mathrm{~s}$ : only the hormone-treated bird produced complete Whistle-shakes, whereas the untreated stimulus male predominantly performed intermediate forms when sprayed with water. Neither of the two stimulus birds performed displays other than (in)complete W histleshakes. Owing to the hormonal implantation, the stimulus males differed in morphology. The testosterone propionate-treated stimulus male had a big cherry-red bill-knob characteristic of adult males in spring which was lacking in the untreated stimulus bird.

All tests were carried out in the same cages, which were about $0.75 \times 1 \times 1.5 \mathrm{~m}$. The test and stimulus birds were transferred from their home cages, described above (experiment I), to the test cages $1 \mathrm{~h}$ before the test started, to give them time to recover from the stress of transport and to become used to the new environment without 


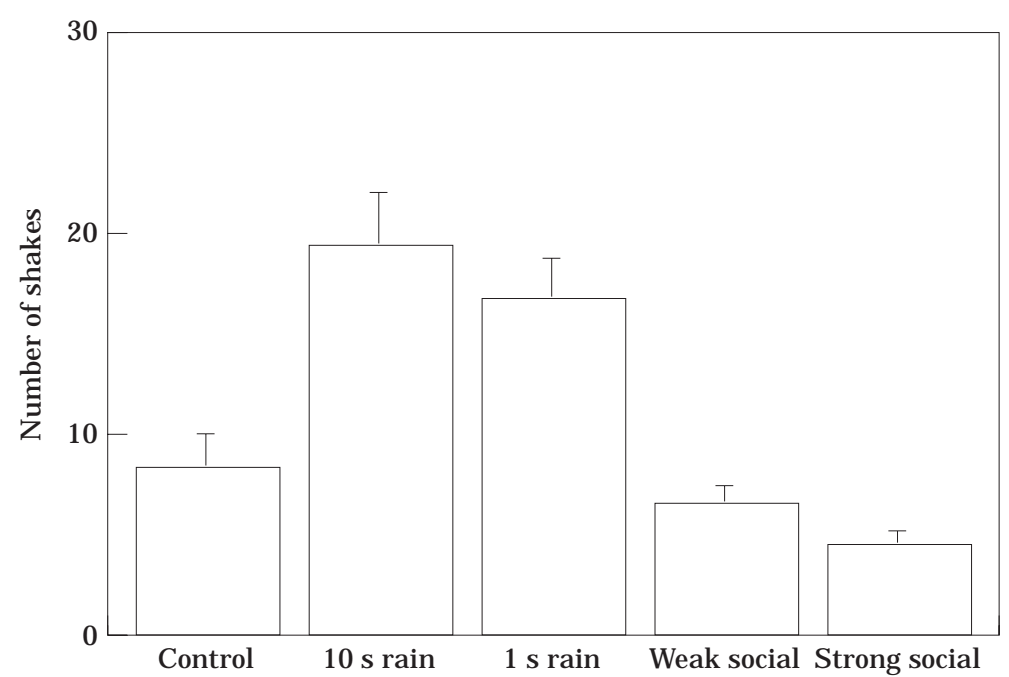

Figure 4. Total number of shakes $(\bar{X}+\mathrm{SE})$ of six male shelducks given as a response to different social and comfort stimuli.

well-known conspecifics. We tested six males, receiving control, comfort and social stimuli. All tests were carried out within 2 weeks. Tests with the same birds were separated by at least 1 day.

We recorded the behaviour of the stimulus and test birds continuously. In the analysis we first $\log (x+1)$ transformed the data and subsequently tested the differences between the stimulus conditions by means of a two-factor MANOVA, in which one factor represented the stimulus condition (five levels, repeated measurement) and the other the form of the shaking pattern (four levels, repeated measurement). Degrees of freedom within cells was 5.

\section{R esults}

A n overall M A N OVA on the frequency of total shaking yielded a significant difference between the five stimulus conditions ( $F$ ig. 4; overall effect of stimulus condition: $\left.\mathrm{F}_{4,20}=6.55, \mathrm{P}<0.05\right)$. A rtificial rain increased the number of shakes (weak comfort stimulus versus control: $t_{5}=7.97, \mathrm{P}<0.01$; strong comfort stimulus versus control: $t_{5}=11.83$, $\mathrm{P}<0.001$ ), in contrast to social stimuli (all Ns). In addition we found a highly significant interaction effect between stimulus condition and form of the shake $\left(F_{12,60}=7.88, P<0.01\right)$. Thus, the different forms of shaking behaviour were affected differently by comfort and social stimuli. Each shaking form had its peak in a different test situation: the Body-shake occurred most often in the $10 \mathrm{~s}$ rain test, the $D$ eep Intermediate form in the $1 \mathrm{~s}$ rain test; $\mathrm{H}$ igh Intermediate forms were observed most often in the weak social stimulus test, whereas Whistle-shakes were predominantly performed in the strong social test (Fig. 5). Post-hoc one-way M A N OVAs, testing the effect of stimulus condition (repeated factor) for each shaking form, revealed significant effects for each of the forms (in all cases, $\mathrm{F}_{4,20}>3.11, \mathrm{P}<0.05$ ).

In conclusion the Whistle-shake was induced by social stimuli and was almost lacking in artificial rain tests, whereas the Body-shake was released by comfort stimuli and inhibited in the social tests. The D eep Intermediate form was sensitive to rain stimuli, whereas the $\mathrm{H}$ igh Intermediate form was elicited by weak social stimuli.

\section{DISCUSSION}

Form analysis suggests that the Whistle-shake display in male shelducks has been phylogenetically derived from the Body-shake, a comfort movement (D üttmann \& G roothuis 1996). Since the Body-shake can easily be manipulated by external stimuli such as artificial rain, the Whistleshake is an excellent model to investigate whether, during the course of evolution, the derived pattern 


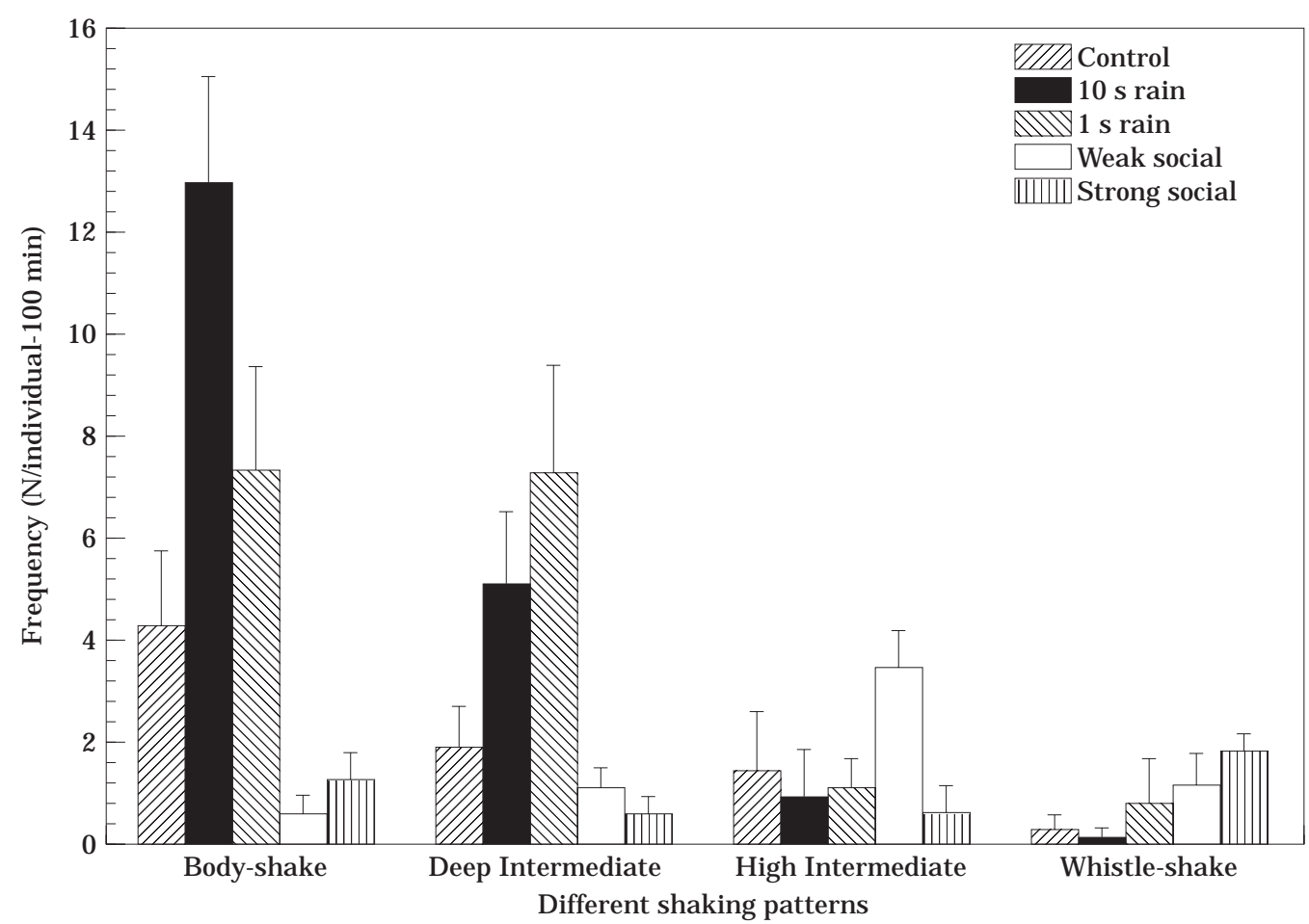

Figure 5. Shaking responses $(\overline{\mathrm{X}}+\mathrm{SE})$ of six male shelducks to different social and comfort stimuli and in a control situation.

has been emancipated from the original causal factors.

In experiment I we showed that in spring socially isolated sheldrakes react exclusively with W histle-shakes when sprayed with water, whereas the same birds in isolation respond to spraying in summer with Body-shakes and Intermediate forms. This confirms the findings of Düttmann \& Groothuis (1996) that this display is not emancipated from the causal factors controlling its presumed evolutionary precursor. In spring, when birds exclusively perform the Whistle-shake, this display is sensitive to social stimulation (D üttmann \& G roothuis 1996). We showed that in summer, when the birds mainly perform the Body-shake, social stimulation neither influenced the frequency of Body-shakes nor induced Whistle-shakes (experiment II). In autumn, when all shaking patterns are present in one bird, artificial rain induced predominantly Body-shakes and D eep Intermediate forms while social stimulation induced almost exclusively Whistle-shakes and $\mathrm{H}$ igh Intermediate forms (experiment III).
The lack of emancipation in the Whistle-shake display is in line with the claim of Baerends (1975) that no good evidence for complete emancipation of social displays exists. In the case of the Whistleshake display, the evolution and the proximate control are much clearer.

\section{C ausation of the Whistle-shake}

O ne may ask whether the Whistle-shake can be released by social stimulation without the presence of comfort stimuli. The results of experiment III suggest that this might not be the case: although social stimulation led to an increase in the performance of Whistle-shakes, the total number of shakes did not change, compared with the control situation. This result suggests that social stimulation is not sufficient to release more shakes than the basal level in the control situation, in which normally occurring comfort stimuli induce a shake from time to time. Social stimulation, as occurred in our experiment III, then only adds the display component, the Bill-tipping with 
trill, to a shake, which was primarily induced by comfort stimuli. However, one could also argue the other way around. The W histle-shake might be primarily induced by the social stimuli of our test. The lack of increase of total shaking might then be explained by the fact that the Whistleshake is equally effective in caring for the plumage as the Body-shake is. Thus there would be no need to give an additional Body-shake after a socially induced Whistle-shake.

Both explanations are valid only when one assumes that vocal stimulation from conspecifics in experiment III did not affect the basal level of shakes in the control situation. This is very likely for two reasons: (1) in playback experiments $\mathrm{H} .-\mathrm{H}$. Bergmann (personal communication) could not find evidence that sheldrakes react to whistling trills of conspecifics; and (2), owing to the season, conspecifics almost never performed Whistle-shakes or other display patterns.

Evidence for the possibility that the Whistleshake can be primarily triggered by social stimuli is given by results of previous experiments. Düttmann \& Groothuis (1996) found an increase in the frequencies of Whistle-shake in spring under the influence of the presentation of a caged intruder. F urthermore, this increase was accompanied by an increase in other social displays, whereas the frequencies of several comfort move ments such as W ing-flapping and Wing-stretching did not change. This indicates that the increase in the $W$ histle-shake in their experiment was not due to an increase in comfort stimuli, for example resulting from body contact with the cage of a caged intruder, but was specifically the result of social stimulation. Why then did we not find an increase in total shakes in our experiment of social stimulation in autumn when all shaking patterns are present in one bird? We can think of three possible explanations: (1) birds in autumn are less sensitive to social stimuli than in spring; (2) birds in autumn, lacking the pronounced bill-knob of birds in spring, were inhibited by the testosteronetreated stimulus bird showing such a cherry-red bill-knob; and (3) our birds were placed in unfamiliar cages, in contrast to the birds in the experiment of D üttmann \& G roothuis (1996).

In contrast to the situation in autumn and spring, social stimulation did not induce any Whistle-shakes in summer when the birds were in moult. This effect of season is likely to depend on the endogenous production of testosterone, which is low during moult, intermediate during autumn and high during the reproductive period in spring ( $R$ atermann 1991). Testosterone might influence the Whistle-shake in at least two ways: (1) it may lower the threshold for the addition of the billtipping with trill component to the Body-shake when the latter is performed because of comfort stimuli and (2) it may increase the impact of social stimuli on the induction of the display. Both possibilities seem to exist. The first is indicated by the finding that in spring (but not in summer) birds reacted to artificial rain with almost exclusively Whistle-shakes even without the presence of social stimuli (experiment I). The second possibility is indicated by the increase in the total number of shakes consisting almost entirely of Whistle-shakes in spring because of social stimulation (D üttmann \& G roothuis 1996).

We used only one stimulus bird in treatments 4 and 5 of experiment III (confrontation with a weak and a strong social stimulus) which might have led to pseudoreplication in the sense that behavioural differences of the test birds in treatments 4 and 5 might be due to differences in some individual characters of the two stimulus birds, and not to differences in testosterone-dependent behaviours. However, the difference in social stimulus between these treatments was very conspicuous; similar but independent experiments revealed similar results ( $G$ roothuis \& D üttmann 1996). F urthermore, in any case the test males reacted on the basis of a difference in a social stimulus which has been the aim of experiment III.

\section{Causation of the Body-shake}

The Body-shake is clearly under the control of comfort factors. In summer, rain stimulation induced Body-shakes in almost $100 \%$ of the tests. F urthermore, the Body-shake is clearly more sensitive to artificial rain than the Whistle-shake: in autumn, rain stimulation induced a clear increase in the frequency of the Body-shake and not in the frequency of the Whistle-shake. We could not find evidence that the Body-shake in males is sensitive to social stimulation (experiments II and III).

In conclusion, the Whistle-shake display is not emancipated from the factor controlling its presumed precursor. The display is, however, sensitive to social stimuli and testosterone, in contrast to the Body-shake. 


\section{ACKNOWLEDGMENTS}

We are greatly indebted to J. P. K ruijt for his support throughout the study. We have benefitted from discussions with $G$. de Vos, A. Ros (G roningen) and H.-H. Bergmann (O snabrück). R. W iegman, S. Veenstra and T. de Boer assisted in taking care of the birds. Comments on the manuscript by $A$. Berglund, J . van R hijn and $M$. $K$ ennedy improved the paper. Financial support was provided by Deutsche Forschungsgemeinschaft (D u 228/1-1) and by Studienstiftung des D eutschen Volkes (Bonn).

\section{REFERE N CES}

Baerends, G. P. 1975. A n evaluation of the conflict hypothesis as an explanatory principle for the evolution of displays. In: Function and Evolution of Behav- iour (Ed. by G. P. Baerends, C. Beer \& A. M anning), pp. 187-227. Oxford: Clarendon Press.

D üttmann, H . 1992. Veränderungen im Individual- und Sozialverhalten während der Jugendentwicklung der Brandente (Tadorna tadorna). Ph.D. thesis, U niversität Osnabrück.

Düttmann, H. \& G roothuis, T. G. G. 1996. Evolutionary origin, proximate causal organization and signal value of the Whistle-shake-display of male shelducks (Tadorna tadorna). Behaviour, 133, 597-618.

$M$ CK inney, F. 1965. The comfort movements of A natidae. Behaviour, 25, 120-220.

Ratermann, M. 1991. J ahreszeitliche Veränderungen von Gestaltmerkmalen und Verhalten vor dem $H$ intergrund hormonaler $F$ aktoren bei der Brandente (Tadorna tadorna). Ph.D. thesis, U niversität Osnabrück.

Ratermann, M., Düttmann, H.\& Bergmann, H.-H . 1990. Jahresperiodik bei der Brandente (Tadorna tadorna): morphologie und verhalten. Proc. Int. 100. D 0-G M eeting, Curr. Topics A vian Biol., 397-403.

Tinbergen, N. 1952. Derived activities: their causation, biological significance, origin and emancipation during evolution. Q. R ev. Biol., 27, 1-32. 